\title{
QT Correction: Using an Observed Regression Factor Applicable to a Population Subset
}

\author{
Charles Oo ${ }^{1}$ and Suraj S. Kalbag ${ }^{2}$ \\ ${ }^{1}$ SunLife Biopharma, Morris Plains, NJ, USA; ${ }^{2}$ Neikalus Communications, Waldwick, NJ, USA \\ Received, October 27, 2015; Revised, November 13, 2015; Accepted, December 8, 2015; Published, January 11, 2016
}

\begin{abstract}
Purpose. A QT interval correction to standardized heart rate (QTc) is essential to compare drug effect or to mitigate cardiac risk in clinical practice. Numerous empirical formulas for QTc have been proposed. However, an effective and readily comprehensible method has been elusive. As QTc is dependent on demographics, concomitant drugs, health status, autonomic and diurnal variation, the applicability of these methods hinge on the characteristics of a population that is assessed. An individual QTc is ideal, but it requires substantial baseline ECG data and is beyond the scope for initial evaluation. As a compromise, an approach for a 'discontinuous' population subset is suggested. In this article, we outline the challenges of QTc, and select a power function $\left[\mathrm{QTc}=\mathrm{QT} /\left\{(\mathrm{RR})^{\alpha}\right\}\right]$ in which a regression factor $\alpha$ relevant to a particular population subset is used. The formula is similar to the one used in the Bazett's $(\alpha=1 / 2)$ or Fridericia's $(\alpha=1 / 3)$ method. The use of this approach is illustrated with two small population subsets separated by age and out- or in-patient status. This QTc approach is relatively simple to implement in drug development or by a busy practitioner within his/her institution. Nevertheless, in view of the limitations of the illustrative sample size and confounding factors of this proposal, additional studies will be necessary for further evaluation of QTc methods.
\end{abstract}

This article is open to POST-PUBLICATION REVIEW. Registered readers (see "For Readers") may comment by clicking on ABSTRACT on the issue's contents page.

\section{INTRODUCTION}

The prevalence of QT interval prolongation, a risk factor for torsade de pointes and death, is high among medical patients due to the presence of QT prolongation drugs, hypokalemia and health deterioration (1). QT interval is inversely related to heart rate (HR), and the correction of QT to standardized HR (QTc; 60 beats/min) is necessary to compare drug effect. Addressing and mitigation of cardiac risk is crucial in drug development and in a clinical practice $(2,3)$. For this purpose, the challenges of QTc are outlined, and a practical approach is proposed as described below.

\section{EMPIRICAL CORRECTIONS}

Over 40 methods have been proposed, and the default is a simple power function $\left[\mathrm{QTc}=\mathrm{QT} /\left\{(\mathrm{RR})^{\alpha}\right\} ; \mathrm{RR}=\right.$ interbeat interval, $\alpha=$ regression factor] derived independently in 1920 by Bazett and Fridericia (4). The Bazett's formula $(\alpha=1 / 2)$ is thought to over-correct at low HR and under-correct at high HR. The Fridericia's formula $(\alpha=1 / 3)$ is believed to be closer to the center of the population distribution and may be used if the HR change is not substantial (5). However, in children, and especially in infants, the average HR could be above 100 beats per minute (versus 60-90 beats per minute in adults), and the Bazett's correction is more appropriate $(6,7)$. In reality, depending on the characteristics of a population subset, the $\alpha$ value could range around $1 / 2$ to $1 / 3(6,8)$.

A perfect correction formula produces a QTc that is completely independent of HR and a plot of QTe versus various HR values should yield a horizontal linear regression line with a slope of zero. A convenient correction will be to correlate the X-axis as HR and the y-axis as QTc, where QTc is computed by using the equation with an observed regression factor $\alpha$ from a plot of QT versus RR. As $\mathrm{HR}$ is inversely related to $\mathrm{RR}$, this approach will nullify the effect of HR on QTc. Nearly all the proposed methods derived their regression factors

Correspondence Author: Charles Oo, SunLife Biopharma, 52 Gatheringhill Court, Morris Plains, NJ 07950, USA. Email: charlesoo@yahoo.com. 
from their respective datasets.

Nonlinear formulas could be more appropriate than linear ones for QTc. The relationship of QT and RR is nonlinear $(5,9)$, and a power format in either the Bazett's or Fridericia's formula could be more applicable (8). Nevertheless, a rich dataset is needed for fitting a nonlinear function, especially at the terminal portions of a QT-RR plot.

\section{QT CORRECTION AND ITS PROPOSED REMEDY}

It is known that the QT-HR relationship is affected by age, sex, health status, physiology and autonomic tone, hysteresis, and diurnal variation. In addition, determining QT interval could be subjective because the $\mathrm{T}$ wave merges gradually with its baseline and the terminal end is not always clearly defined (10). Due to high inter- and intrasubject variabilities, frequent false findings have been reported $(5,11)$. With the low signal-to-noise ratio, there may be no readily interpretable relationship for some drugs that affect HR. A consistent method of acquiring RR data directly (not calculated from HR) will be essential. In addition, to reduce the effects of hysteresis and diurnal variation, a selective data acquisition at steady-state and at constant times of day will be necessary.

All standard formulas assume consistent shapes at all times in the QT-HR relationship, and this assumption may not apply to patients with disease or on drugs (5). In fact, the ICH E14 guidance allows for the flexible inclusion of other correction methods, in addition to submitting the uncorrected QT and RR data, and those for the Bazett's and Fridericia's formulas (12). Therefore, any unexpected QTc value should not be ignored as an outlier but will need to be evaluated for a possible aberrant trend.

Ideally, QT correction should be performed on an individual basis (13), but a very large number of QT-HR pairs at baseline are required. A continuous 12-lead Holter monitor, or a 1-stage population PK-PD analysis where HR can be set as a covariate will be necessary $(8,14)$. In addition, QTc by standard methods does not account for the changes in autonomic tone or QT-RR hysteresis, and a dynamic beat-to-beat QT value that defines the entire RR range without the use of a correction function could be needed (15). However, these approaches are more sophisticated than are necessary for an early evaluation of QT prolongation, while the main purpose is to decide whether a new drug can be developed or whether additional follow-up is warranted.

Instead of individual QTc, a 'discontinuous' approach, where the whole population is differentiated into more homogeneous subsets, is proposed. Various variables such as demographics (e.g., age, sex) and health status (e.g., out- or inpatient, specific diseases) can be used as differentiators into population subsets. The choice is dependent on the needs and availability of these variables.

\section{DATA ILLUSTRATION}

Among the confounding factors, aging is known to affect QT with its accompanying cardiac hypertrophy, prolongation of myocardial action potential, and deterioration of health (16). For illustration, a limited data are presented using age and out- or in-patient status as differentiators for the two observed population subsets: (a) 284 pairs of QT-HR data from 106 young out-patients (104 males, 2 females; mean $[ \pm \mathrm{SD}]$ age $29.7 \pm 2.7$ years), and (b) 1151 pairs of QT-HR data from 105 old inpatients (58 males, 47 females; mean $[ \pm \mathrm{SD}]$ age $66.9 \pm 12.8$ years). Presumably, more drugs were onboard for in-patients with their worsening health statuses as compared to those out-patients. For both subsets, the uncorrected QT intervals inversely decrease with HR (Fig. 4). To derive appropriate corrections, the mean observed regression factors $(\alpha)$ of the power functions are obtained for the young out-patients $(=0.317)$ and old in-patients $(=0.394)$ subsets (Fig. 1). By using these two regression factors (dissimilar at different institutions and population subsets) and without separating the model building and test datasets, the resultant institutional QTc means will be independent of HR (Fig. 2). Based on the linear trendlines and equations, the empirically fixed factors used in the Bazett's and the Fridericia's methods, and the linear Framingham's method $\{\mathrm{QTc}=\mathrm{QT}+0.154 *(1-\mathrm{RR})\}$ may not correct as well (Fig. 4).

As mentioned above, other variables could be included for QTc evaluation, such as sex difference. Nevertheless, the effect of sex on QTc could be 
controversial. Contrary to popular belief, sex could

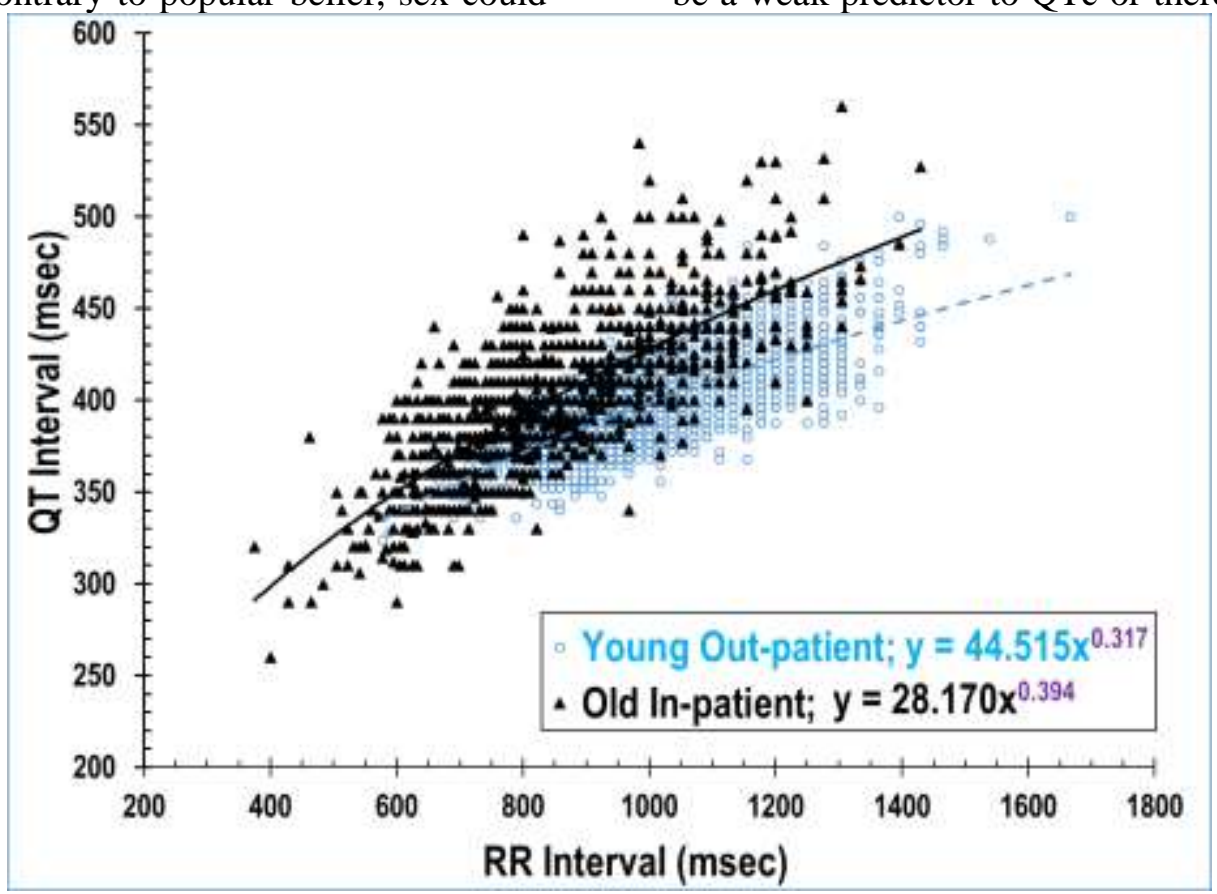

Figure 1: The plots of QT versus RR in a power function $Q T=\beta(R R)^{\alpha}$ to derive the observed regression factor $\alpha$ for the young out-patient and old in-patient subsets in the illustrative dataset, where $\beta$ is a constant. Trendlines and equations of the power functions are shown for the two population subsets.

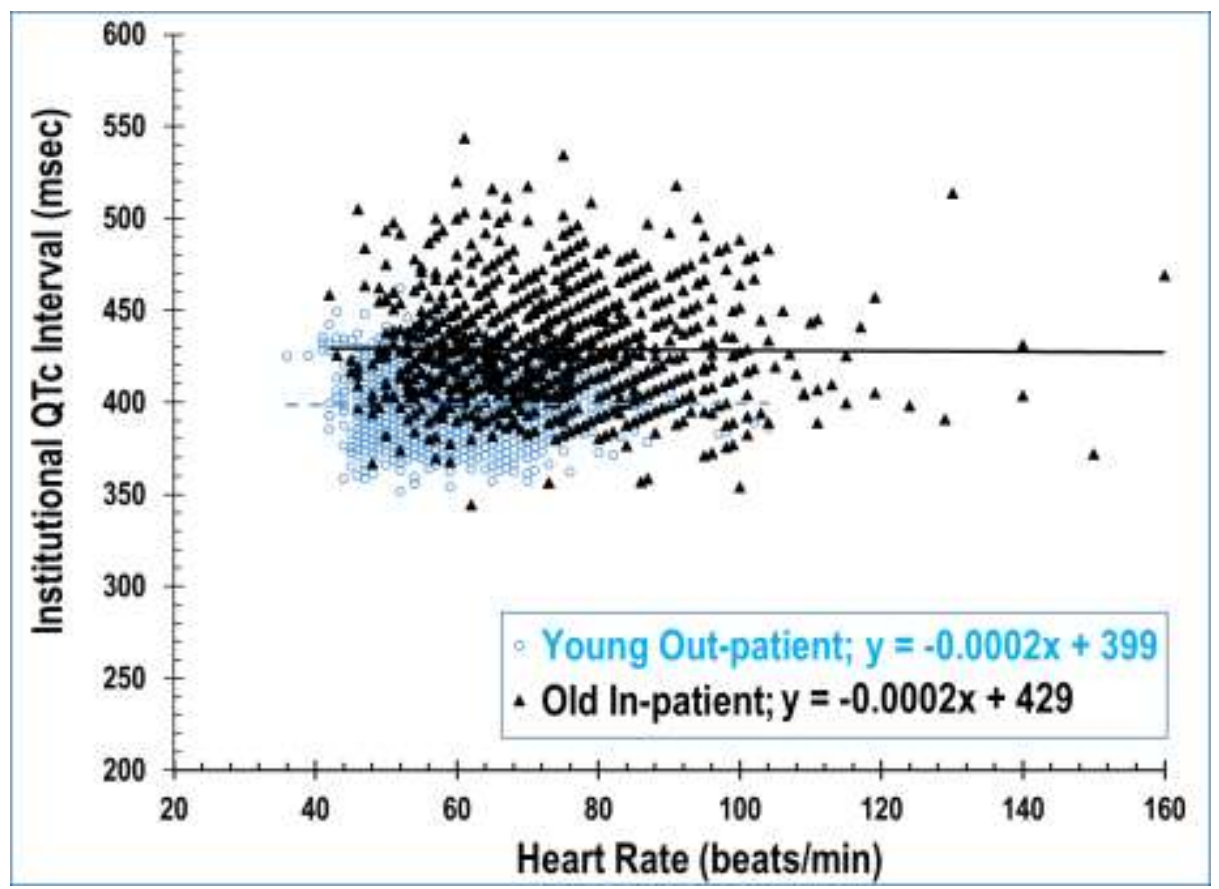

Figure 2: The plots of the Institutional QTc versus heart rate of a power function $Q T c=Q T /\left\{(R R)^{\alpha}\right\}$ using the observed regression factors $(\alpha)$ for the young out-patients $(=0.317)$ and old in-patients $(=0.394)$ subsets in the illustrative dataset. Linear trendlines and equations are shown for the two population subsets. 
longer QTc in a woman than in man (16). This is particularly so in older women, presumably with the decrease in female hormones (16). For this illustrative subset of the old in-patients where the numbers for males and females are relatively balanced, there is no dispersion difference between the two sexes based on QT-RR plots (Fig. 3). No sex assessment is conducted in the young outpatient subset due to limited number for females.

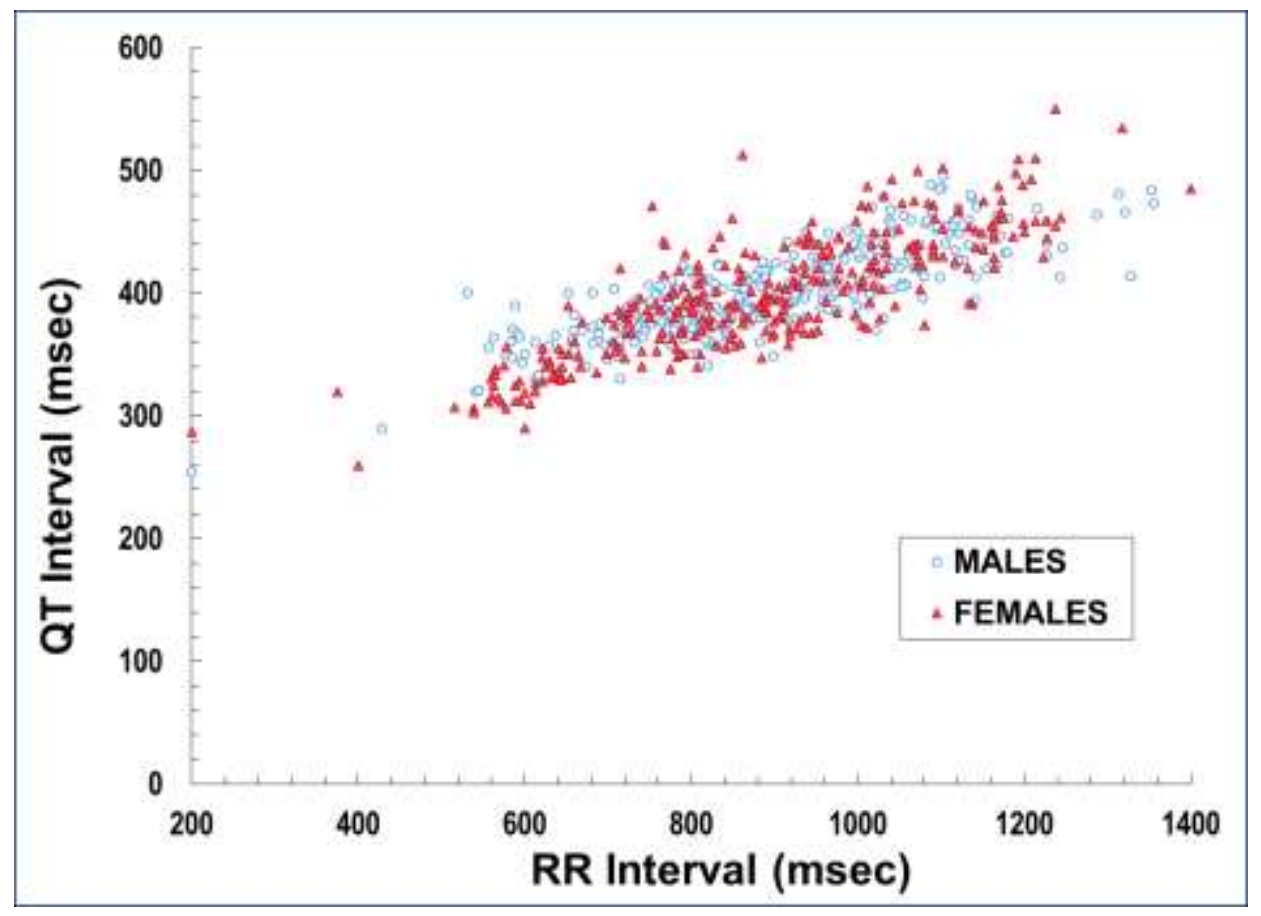

Figure 3: The plots of QT versus RR intervals for males and females in the old in-patient subset

No systematic comparison is conducted for this limited dataset. It is anticipated that with the accumulation of data of sufficient quantity and quality, an improvement in regression factors will be obtained to capture the characteristics of population subsets. Bear in mind that both the Bazett's and Fridericia's formulas use small sample sizes of 39 and 50, respectively (17), while the commonly used Framingham's formula is derived from about 5000 patients (18). However, as mentioned above, a power format could be preferred over a linear function.

\section{CONCLUSION}

With the accumulation of demographic, health status, and ECG data to derive the regression factors for specific population subsets, this institutional correction approach is relatively simple to implement in early stage of drug development or by a busy practitioner. Nevertheless, in view of the limitations of the illustrative sample size and confounding factors of this proposal, additional studies will be necessary for further evaluation of QTc methods. 


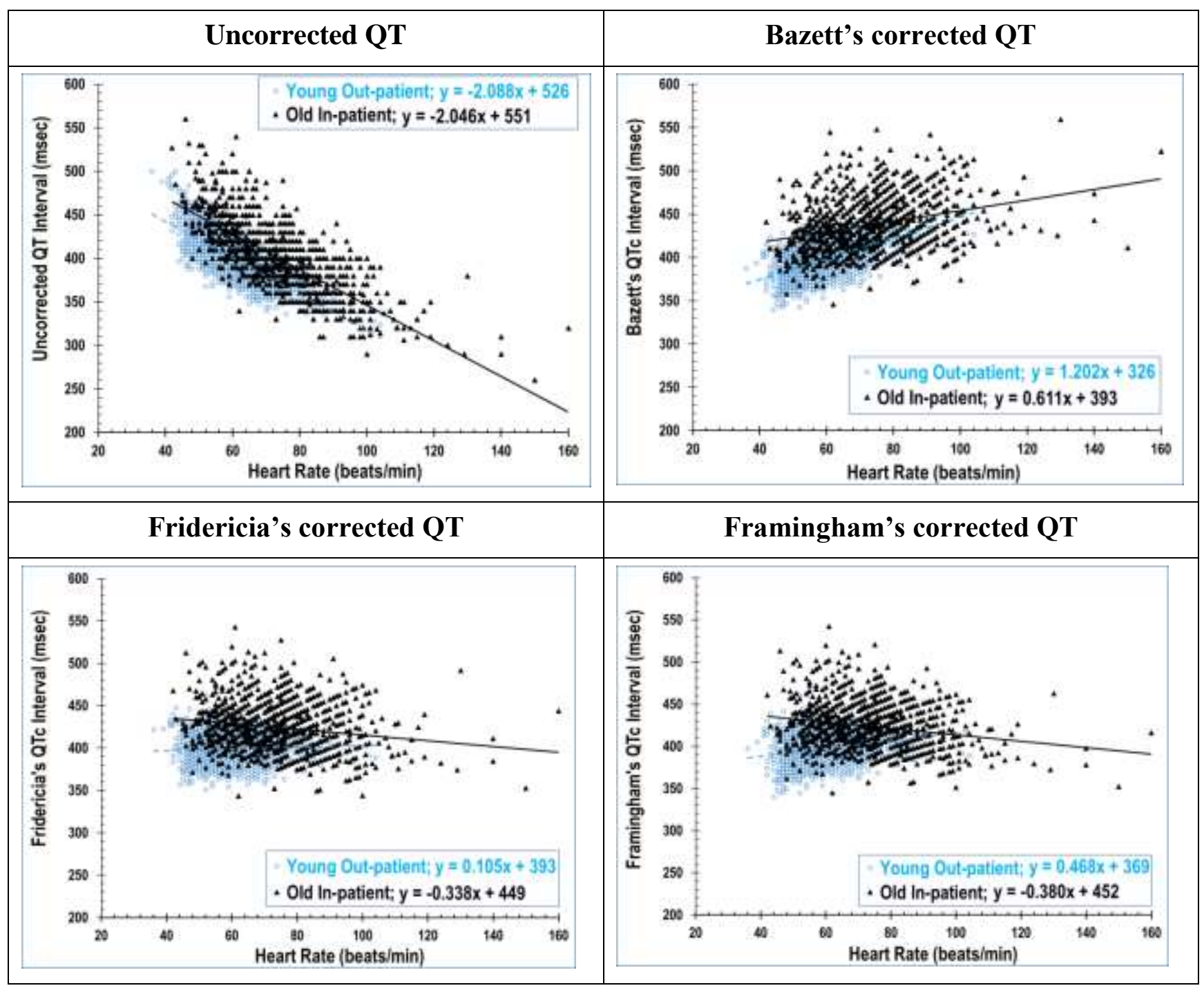

Figure 4: Plots of the uncorrected, Bazett's corrected, Fridericia's and Framingham's corrected QT versus heart rate for the young out-patient and old in-patient subsets. Linear trendlines and equations are shown for the two populations.

\section{REFERENCES}

1. Pasquier M, Pantet O, Hugli O, Pruvot E, Buclin T, Waeber G, et al. Prevalence and determinants of QT interval prolongation in medical inpatients. Intern Med J. 2012;42(8):933-940.

2. Crouch MA, Limon L, Cassano AT. Clinical relevance and management of drug-related QT interval prolongation. Pharmacotherapy. 2003;23(7):881-908.

3. Cheng JWM, Dopp AL, Kalus JS, Rogers KC, Sanoski CA, White CM. Key articles and guidelines in the management ofarrhythmias, 2004-2010. Pharmacotherapy. 2011;31(1):1e - 32e.
4. Moss AJ. Introductory note to a translated classic article of L. S. Fridericia. Ann Noninvasive Electrocardiol. 2003;8(4):341-342.

5. Garnett CE, Zhu H, Malik M, Fossa AA, Zhang J, Badilini F, et al. Methodologies to characterize the QT/corrected QT interval in the presence of druginduced heart rate changes or other autonomic effects. Am Heart J. 2012;163(6):912-930.

6. Phan DQ, Silka MJ, Lan YT, Chang RK. Comparison of formulas for calculation of the corrected QT interval in infants and young children. J Pediatr. 2015 Apr;166(4):960-4.e1-2.

7. Qiu H, Bird GL, Qu L, Vetter VL, White PS. 
Evaluation of QT interval correction methods in normal pediatric resting ECGs. Comput Cardiol. 2007;34:431-434.

8. Zheng M, Burt D, Chan W, Hawthorne D, Gasior M, Bhagwagar Z, et al. Comparison of different QT interval correction methods for heart rate and QT beat-to-beat method in a thorough QT study of a triple monoamine reuptake inhibitor BMS-820836. J Clin Pharmacol. 2015;55(10):1137-1146.

9. Malik M, Hnatkova K, Kowalski D, Keirns JJ, van Gelderen EM. QT/RR curvatures in healthy subjects: sex differences and covariates. Am J Physiol Hear Circ Physiol. 2013;305(12):H1798H1806.

10. Postema PG, Wilde AAM. The measurement of the QT interval. Curr Cardiol Rev. 2014;10(3):287-294.

11. Hutmacher MM, Chapel S, Agin MA, Fleishaker JC, Lalonde RL. Performance Characteristics for Some Typical QT Study Designs Under the ICH E-14 Guidance. J Clin Pharmacol. 2008;48(2):215-224.

12. Guidance for Industry; E14 Clinical evaluation of QT/QTc interval prolongation and proarrhythmic potential for non-antiarrhythmic drugs, 2005. U.S. Department of Health and Human Services Food and Drug Administration. Available from: http://www.fda.gov/cber/guidelines.htm

13. Malik M, Färbom P, Batchvarov V, Hnatkova K, Camm AJ. Relation between QT and RR intervals is highly individual among healthy subjects: implications for heart rate correction of the QT interval. Heart. 2002;87(3):220-228.

14. Piotrovsky V. Pharmacokinetic-pharmacodynamic modeling in the data analysis and interpretation of drug-induced QT/QTc prolongation. AAPS J. 2005;7(3):E609-E624.

15. Fossa AA, Zhou M. Assessing QT prolongation and electrocardiography restitution using a beat-to-beat method. Cardiol J. 2010;17(3):230-243.

16. Mangoni AA, Kinirons MT, Swift CG, Jackson SH. Impact of age on QT interval and QT dispersion in healthy subjects: a regression analysis. Age Ageing. 2003;32(3):326-331.

17. Rabkin SW, Cheng XB. Nomenclature, categorization and usage of formulae to adjust QT interval for heart rate. World $\mathrm{J}$ Cardiol. 2015;7(6):315-325.

18. Sagie A, Larson MG, Goldberg RJ, Bengtson JR, Levy D. An improved method for adjusting the QT interval for heart rate (the Framingham Heart Study). Am J Cardiol. 1992;70(7):797-801. 